\title{
Scientific Workflow Infrastructure for Computational Chemistry on the Grid
}

\author{
Wibke Sudholt ${ }^{1}$, Ilkay Altintas ${ }^{2}$, and Kim Baldridge ${ }^{1,2}$ \\ ${ }^{1}$ Institute of Organic Chemistry, University of Zurich, Winterthurerstrasse 190, \\ $\mathrm{CH}-8057$ Zurich, Switzerland \\ \{wibke, kimb\}@oci.unizh.ch \\ ${ }^{2}$ San Diego Supercomputer Center (SDSC), UC San Diego, 9500 Gilman Drive, \\ La Jolla, CA 92093-0505, USA \\ altintasesdsc.edu
}

\begin{abstract}
We present ongoing research in the Resurgence (RESearch sURGe ENabled by CyberinfrastructurE) project. This infrastructure shall enable the flexible combination of computational chemistry tools from a unified interface, with the focus on automated high-throughput processing. The implementation is based on the idea that the time-consuming parts of the calculations can be distributed onto computational Grids using the Kepler scientific workflow system and the Nimrod toolkit for distributed parametric modeling. We describe an example workflow that allows preparing, running, and displaying jobs on different molecules, employing the GAMESS quantum chemical program package.
\end{abstract}

\section{Introduction}

\subsection{Scientific Background}

Catalyzed by life and material sciences, studying vast amounts of molecules becomes more and more important in applied chemical research. In the drug discovery pipelines of pharmaceutical companies, many thousand compounds are screened for activity in a high-throughput manner, often using computational chemistry approaches such as QSAR (Quantitative Structure Activity Relationships) and docking. Fundamental chemistry research, on the other hand, has traditionally been sparsely automated. However, established computational chemistry tools such as quantum chemistry, molecular dynamics, and continuum electrostatics nowadays allow the accurate prediction of molecular properties. Advances in technology continuously speed up these calculations. Together with the opportunities of data mining, one hopes to learn new chemical concepts from analyzing many molecular computations.

Moreover, driven by the interest in large and complex systems such as biomolecules, solutions, and materials, it is increasingly necessary to couple different computational chemistry packages. Algorithms from various scientific domains are combined, and resolutions range over several time and length scales, an important example being the QM/MM (Quantum Mechanics/Molecular Mechanics) techniques. However, these studies usually require the direct integration of application codes. The 
purpose of the Resurgence project is thus the flexible and reusable coupling of computational chemistry packages. This will allow easier exchange of data between applications to realize new scientific ideas, and building of pipelines for automation of tasks. Our focus is on calculations for different compounds or conformers.

\subsection{Distributed Scientific Computing}

The underlying physical equations often make computational chemistry calculations very compute- and data-intensive. Complexity of the parallelized codes often results in highly communication-dependent algorithms. However, technology moves to loosely-coupled Grid infrastructures. Computational Grids are defined as "a hardware and software infrastructure that provides dependable, consistent, pervasive, and inexpensive access to high-end computational capabilities" [1]. Virtual organizations manage the contribution and distribution of resources on a Grid. Established Grid projects have created new opportunities for computational research.

Unfortunately, apart from a few examples (e.g., [2, 3, 4]), building and using Cyberinfrastructure for computational chemistry has been lagging behind the hype. Not many applications have been ported to a Grid infrastructure, exceptions being for example the OpenMolGRID [5] and Gemstone [6]. Due to the long runtimes and high resource demands of the calculations, service-oriented architectures are seldom applied. Computational chemistry programs often result from years of collaboration. To gain speed, they are mainly written in procedural programming languages such as Fortran or $\mathrm{C}$, using formatted input and output file formats specific to each code. Many programs are commercial, and only available as binaries or in-house. This makes it difficult to adapt computational chemistry packages to the requirements of Grid computing. But also Grid computing itself has partly stayed behind its promises. Most Grid middleware has long been in development state and exposes APIs complex to work with for non-specialists. Many management and policy issues have to be solved before a Grid infrastructure can be set up, which is often only accessible for selected researchers. Finally, network latency over long distances, hardware and software heterogeneity, and missing control on resource availability in Grids make it less efficient to port the highly-coupled parallel computational chemistry programs.

Nevertheless, Grid computing has a lot to offer for computational chemistry. Neighboring disciplines such as bioinformatics and high-energy physics with similar problems, though stronger orientation towards web services and data Grids, respectively, have demonstrated the benefits of using such infrastructures. Embarrassingly parallel calculations such as parameter sweeps and optimizations are perfectly suited for running on Grids. Corresponding tools such as Nimrod [7] and APST [8] provide wrappers around existing codes to distribute them over a number of machines, and have been successfully applied in scientific studies. What these tools are missing though are simple ways to seamlessly couple different applications to form pipelines, where the results of one stepare used as basis for the next. The purpose of the Resurgence project is thus to provide a workflow tool that allows to flexibly combine computational chemistry codes within one common interface. This should be easy to use for beginners, hiding the complexity of the middleware, but details should be accessible for advanced users. Compute-intensive steps should be distributed onto the Grid, while short and interactive steps should stay on the local machine. 


\subsection{Scientific Workflow Systems}

As the scientific process moves into an era of distributed data and computation, the needs of scientific studies have also changed. Now, the solution to a scientific problem often involves reaching out to Grids, discovering datasets, running pipelined and repeated analysis tasks in parameterized calculations, storing the outcomes into databases, registering the workflows and results into online portals, and visually displaying them. Today's scientists are expected to use all these technologies.

Scientific workflows emerged as a glue to compose different technologies by providing interfaces to run a coordinated set of data and computation components in a systematic and automated way. They can be applied to combine data integration, analysis, and visualization steps into larger 'knowledge discovery pipelines' and 'Grid workflows' [9]. Scientific workflow research and system development have recently been the focus of several big efforts. However, the lack of generic graphical interfaces that abstract the technical details from users and let them focus on the science, has been a major obstacle for using traditional workflow systems rather than customized applications. Scientific workflow user environments, for example Kepler [10], Taverna [11], and Triana [12], aim at improving this situation by 'wrapping' Grid tools and making them available in a user-friendly visual programming environment.

Kepler is particularly suited for computational chemistry workflows, as it does not only provide web service, Grid, and XML-based features, but also command-line wrapping, file system interaction, and string processing capabilities. The rest of this paper describes our case study in using Kepler for a computational chemistry pipeline. We first explain the requirements of the scientific problem and how different tools were used together to solve it. Then we describe how we utilized Kepler's components to assemble the workflow. Finally, we discuss the results and unresolved issues.

\section{Tools}

\subsection{Computational Chemistry Programs}

In the first Resurgence [13] implementation, we concentrate on the preparation, execution, and analysis of quantum chemical calculations on a number of small molecules. Our main principles are the restriction to open source codes to retain the accessibility for all users, and the use of downloadable versions of all application programs without modifications. The core tool employed is the GAMESS ab initio quantum chemistry package [14]. It is a well-established open source code to determine chemical properties based on the Schroedinger equation. It is mainly written in Fortran, highly parallelized, and can run very efficiently on different computer architectures. As example of an analysis tool for the calculation results we selected the molecular graphics program QMView [15]. It allows displaying and modifying the threedimensional structures of chemical compounds and provides a large variety of options to examine their molecular properties. An important issue in computational chemistry studies is related to file formats and their transformation. Despite emerging standards such CML (Chemical Markup Language) [16], the vast majority of applications still use their own legacy input and output file formats. This is one of the main limiting 
factors of program interoperability. Therefore, we included two key file format conversion tools, Open Babel [17] and its predecessor Babel [18].

\subsection{Nimrod Distributed Parametric Modeling Toolkit}

As Grid computing interface for the parameterized calculation jobs, Nimrod/G [7] is used. It is part of the Nimrod toolkit for distributed parametric modeling and was already successfully employed previously [2]. Nimrod can perform embarrassingly parallel parameter sweeps (Nimrod/G) and optimizations (Nimrod/O) distributed on a Computational Grid. The user specifies a list of available computer resources, and for each computational 'experiment' (i.e., collection of parallel jobs), selects the particular resources to use. A short, declarative 'plan file' describes the parameters to consider and the tasks to be performed for each single job. Nimrod then allocates, executes, controls, and collects the individual jobs via 'agents' submitted automatically to the involved resources. Nimrod only has to be installed once on the central root node, and then communicates with the Grid nodes via the corresponding middleware, mainly the Globus toolkit [19]. At the backend of Nimrod is a PostgreSQL database and Python code. It has a command-line interface, which we employ here, a web portal (Nimrod Portal), and an Excel interface (Active Sheets).

\subsection{Kepler Workflow Approach}

We have selected Kepler [10] as the environment to utilize the above-mentioned tools in a workflow. Kepler is a collaboration between several domain research projects, to develop an extensible and customizable open-source scientific workflow system. The Kepler system allows scientists from different disciplines (bioinformatics, cheminformatics, ecoinformatics, geoinformatics, oceanography, astrophysics, etc.) to design and execute scientific workflows. It builds on top of the mature Ptolemy II software developed at UC Berkley [20]. Ptolemy II is a Java-based system along with a set of APIs for heterogeneous hierarchical modeling. The focus of Ptolemy II is to build models based on the composition of existing components, which are called 'actors' Actors are encapsulations of parameterized actions performed on input data to produce output data. Inputs and outputs are communicated through ports within the actors. The interaction between the actors is defined by Models of Computation (MoC). A MoC specifies the communication semantics among ports and the flow of control and data among actors. Directors are responsible for implementing particular MoCs, and thus define the 'orchestration semantics' for workflows. By changing the director, one can change the scheduling and execution semantics of a workflow, without changing any of the components or the network topology. An example for a director is the 'Synchronous Data Flow (SDF)' domain, in which a sequential execution order of actors can be statically determined prior to execution. Here we need to combine it with the more dynamic 'process networks (PN)' director, which also demonstrates the usage of multiple execution domains in one workflow. The descriptions of Kepler workflows are saved in an XML-based format called Modeling Markup Language (MoML). MoML is the primary persistent file format for Ptolemy II models and Kepler workflows. It also represents the primary mechanism for constructing workflows whose definition and execution is distributed on the network. 


\section{Implementation and Results}

Our initial case study tests the capabilities of Kepler, and the applicability of Kepler workflow development methodologies to computational chemistry calculations and the spreading of tasks onto distributed nodes. The conceptual workflow underlying our example implementation is simple (see Figure 1). Several files containing the atomic coordinates of different molecules are provided. From these, input files for the quantum chemical program package GAMESS are generated. Then the corresponding calculations are executed. Finally, the results of the computations are visualized using the QMView software. A pipeline like this is part of the daily work of computational chemists. The largest variant is usually that different molecular systems, application codes, and keyword and parameter settings are used for the preparation, calculation and visualization steps. If more than a few compounds have to be processed, the typical practice is to use shell scripts. The Resurgence implementation into Kepler provides an easier, more flexible, and reusable manner to accomplish the same tasks.

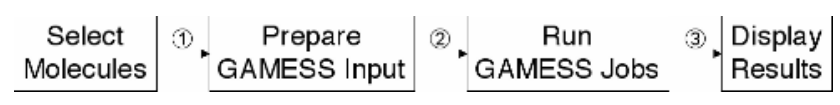

Fig. 1. Conceptual workflow

However, it is clear that the workflow in Figure 1 cannot be directly realized, as different file formats are involved. In our example, we start with CML files, which are then transformed into GAMESS input files, processed into GAMESS output files, and finally converted into PDB (Protein Data Bank) [21] files, which can be displayed. Other format transformations would be possible. This means that at steps number 1 and 3, additional format conversion programs have to be included, in our case Open Babel and Babel. Moreover, the GAMESS calculations are very resource-intensive and time-consuming, and thus the most interesting step to target for Grid distribution. On the other hand, the graphical display and the data-intensive, but fast file format transformations are currently better performed on the local machine. However, if management and storage requirements for these steps increase in future applications, database or data Grid capabilities already available in Kepler might be employed.

The major Kepler actors used for the construction of the executable workflow for the mentioned conceptual workflow are the CommandLine and ExternalExecution actors as well as generic textual display and various constant, array, string function, and file system manipulation actors. The CommandLine and ExternalExecution actors are wrappers for executing commands that run legacy code in a workflow. Their purpose here is to call the diverse employed external programs or corresponding automatically generated temporary shell-script wrappers. To conceptually organize the workflow and to be as modular as possible, we have extensively used composite actors and MoML actor classes. Composite actors are combinations of other actors that are implemented as a block diagram and can be saved into the actor library for reusing and updating purposes. Actor classes allow applying these composite entities in workflows in a similar way as one can use native atomic actor Java classes. 


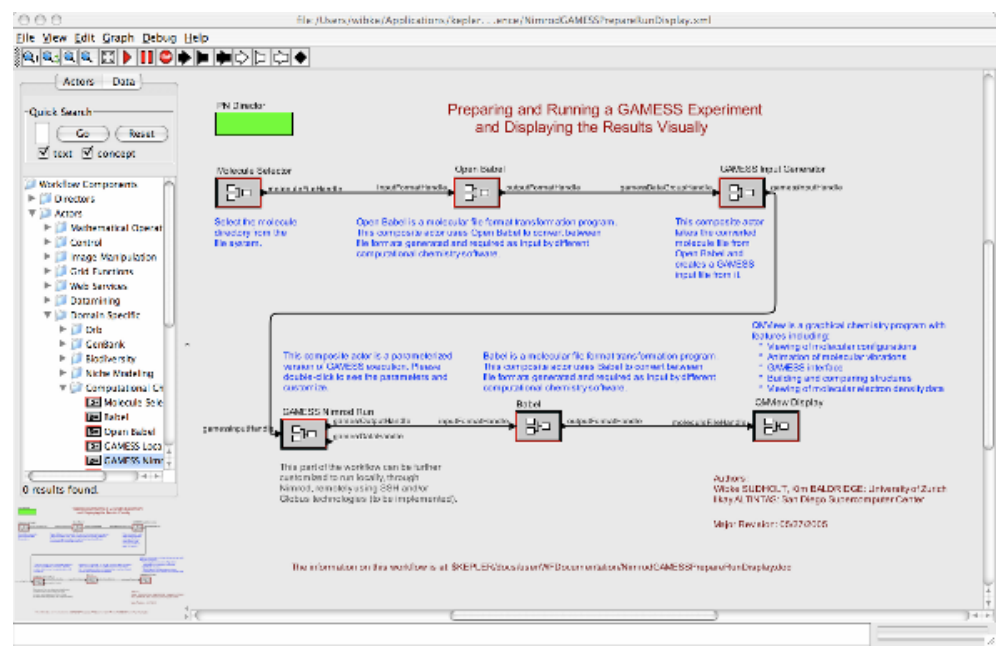

Fig. 2. GAMESS/Nimrod workflow in Kepler

Figure 2 illustrates the Kepler view for the workflow that implements the conceptual workflow in Figure 1. The constructs that make up the workflow were abstracted to six main steps: 'Molecule Selector' to choose the input files for a set of target molecules, 'Open Babel' to transform these files into the GAMESS input format, 'GAMESS Input Generator' to annotate the inputs with the right set of calculation keywords, 'GAMESS Nimrod Run' for the Grid execution, 'Babel' for transformation of GAMESS outputs to PDB format, and finally, 'QMView Display' to visualize the outputs for each molecule. The only data sent between these actors are sequences of file handles belonging to the individual molecules. The corresponding file name bases also serve as the 'parameter' in the auto-generated intermediate Nimrod/G plan file. On this highest-level view of the workflow, the most important variables of the computations can be specified. However, all described steps are composite actor classes which have several layers of sub-workflows hidden inside. By opening and modifying them, the user can fine-tune the calculation pipeline to various degrees. We have tested the workflow for geometry optimizations of a number of different small molecules on a Mac laptop for local execution. For distributed Nimrod execution, a small Globus-based Grid consisting of two seven and eight-node Xeon Linux clusters can be used. Results from ongoing tests will be made available online.

\section{Discussion and Conclusions}

The presented example workflow allows to easily setup a pipeline to prepare, run, and display quantum chemical calculations on different compounds. The compute-intensive part may be distributed onto a computational Grid with the help of the Nimrod toolkit, reducing the time for this embarrassingly parallel step. Due to the graphical visualization of the results at the end, the number of molecules that can be treated concurrently is limited. The most important parameters, such as file types, directory names, external binary locations and arguments, important GAMESS keywords, 
as well as the experiment name, can be specified on the highest workflow level. Tuning of further details of the calculations, for example seldom used GAMESS keywords and Grid node set up can be modified using sub-components of composite actor classes. Due to its high modularity, each component can be reused in other workflows. The GAMESS Nimrod execution actor can substituted by a GAMESS local execution actor without changing the rest of the pipeline. Overall, our implementation is expected to enhance the design and execution of computational chemistry pipelines. By reducing complexity and increasing manageability, adaptability, and reusability as well as by transparent access to Grid resources, preparation and calculation time and effort decreases and more advanced workflows are possible. However, success will finally be measured by its usage scientific studies.

Despite this progress, we did not reach all our goals yet. The integration of the external application codes GAMESS, QMView, Open Babel, and Babel is already far developed. However, the implementation of Nimrod/G currently only covers a part of its functionality. In addition to Nimrod and local execution, implementing $\mathrm{SSH}$, Globus, and web service capabilities for GAMESS is planned. Certainly, the Resurgence interface should be expanded to further domain-specific computation codes. However, also some fundamental aspects of our solution need to be looked at. Its robustness in real high-throughput settings remains to be tested. The necessary file format transformations could benefit from a systematic and rigorous typing system. And finally, the logging, monitoring, and control of the long-running computational experiments have to be improved. The Resurgence module of the Kepler scientific workflow system can be downloaded free of charge from the Kepler website and CVS.

\section{Acknowledgements}

We thank Celine Amoreira and Yohann Potier from the University of Zurich, Adam Birnbaum and Jerry Greenberg from SDSC, Yang Zhao from UC Berkeley, and David Abramson and his group from Monash University, Australia, for collaboration. W.S. acknowledges support from the CoLab at ETH Zurich, K.B. from the NSF-NMI (ANI-0223043), NSF-PRAGMA, and NIH-NCRR (NBCR-RR08605).

\section{References}

[1] Foster, I., Kesselman, C.: Computational Grids. In I. Foster and C. Kesselman, eds., The Grid: Blueprint for a New Computing Infrastructure, Morgan Kaufman(1999), pp. 15-51.

[2] Sudholt, W., Baldridge, K.K., Abramson, D., Enticott, C., Garic, S., Kondric, C., Nguyen, D.: Application of grid computing to parameter sweeps and optimizations in molecular modeling. Future Generation Computer Systems, 21 (2005), pp. 27-35.

[3] Baldridge, K.K., Greenberg, J.P., Sudholt, W., Mock, S., Altintas, I., Amoreira, C., Potier, Y., Birnbaum, A., Bhatia, K., Taufer, M.,: The Computational Chemistry Prototyping Environment, Proceedings of the IEEE, 93 (2005), pp. 510-521;

[4] Baldridge, K.K., Sudholt, W., Greenberg, J.P., Amoreira, C., Potier, Y., Altintas, I., Birnbaum, A., Abramson, D., Enticott, C., Garic, S.: Cluster and Grid Infrastructure for Computational Chemistry and Biochemistry. In A.Y. Zomaya, ed., Parallel Computing for Bioinformatics and Computational Biology, Wiley (2005), pp. 533-552. 
[5] http://www.openmolgrid.org/.

[6] http://www.baldridge.unizh.ch/gemstone/.

[7] Abramson, D., Giddy, J., Kotler, L.: High Performance Parametric Modeling with Nimrod/G: Killer Application for the Global Grid?. International Parallel and Distributed Processing Symposium (IPDPS), Cancun, Mexico (2000), pp. 520-528; http://www.csse.monash.edu.au/ davida/nimrod/.

[8] http://grail.sdsc.edu/projects/apst/.

[9] Altintas, I., Birnbaum, A., Baldridge, K.K., Sudholt, W., Miller, M., Amoreira, C., Potier, Y., Ludaescher, B.: A Framework for the Design and Reuse of Grid Workflows. Scientific Applications of Grid Computing: First International Workshop, SAG 2004, Beijing, China, LNCS, 3458 (2004), pp. 119-132.

[10] Ludaescher, B., Altintas, I., Berkley, C., Higgins, D., Jaeger-Frank, E., Jones, M., Lee, E., Tao, J., Zhao, Y.: Scientific Workflow Management and the Kepler System. Concurrency and Computation: Practice \& Experience, Special Issue on Scientific Workflows (2005), to appear; http://www.kepler-project.org/.

[11] http://taverna.sourceforge.net/.

[12] http://www.trianacode.org/.

[13] http://www.baldridge.unizh.ch/resurgence/.

[14] Schmidt, M.W., Baldridge, K.K., Boatz, J.A., Elbert, S.T., Gordon, M.S., Jensen, J.H., Koeski, S., Matsunaga, N., Nguyen, K.A., Su, S.J., Windus, T.L., Dupuis, M., Montgomery, J.A.: The General Atomic and Molecular Electronic Structure System. J. Comput. Chem., 14 (1993), pp. 1347-1363; http://www.msg.ameslab.gov/GAMESS/.

[15] Baldridge, K.K., Greenberg, J.P.: QMView: A computational chemistry threedimensional visualization tool at the interface between molecules and mankind. J. Mol. Graphics, 13 (1995), pp. 63-66; http://www.nbcr.net/software/QMView/.

[16] http://www.xml-cml.org/; http://cml.sourceforge.net/.

[17] http://openbabel.sourceforge.net/.

[18] http://www.ccl.net/cca/software/UNIX/babel/.

[19] http://www.globus.org/.

[20] http://ptolemy.eecs.berkeley.edu/ptolemyII/.

[21] http://www.rcsb.org/pdb/. 